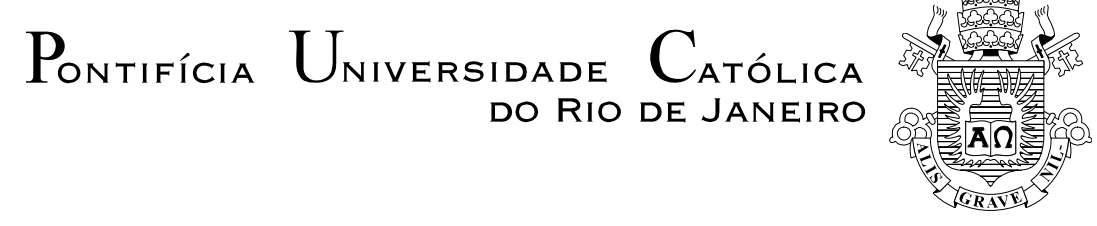

Henrique Bastos Graciosa

\title{
A Transformação das Redes Ópticas no Acesso e no Anel Backbone Metropolitano: Alternativas Tecnológicas, Econômicas e Novos Serviços
}

\author{
Dissertação de Mestrado
}

\begin{abstract}
Dissertação apresentada ao Programa de PósGraduação em Engenharia Elétrica da PUC-Rio como requisito parcial para obtenção do título de Mestre em Engenharia Elétrica.
\end{abstract}

Orientador: Prof. Marbey Manhães Mosso

Rio de Janeiro

Janeiro de 2012 


\section{Pontifícia Universidade C atólica $_{\text {a }}$ \\ DO RIO DE JANEIRO

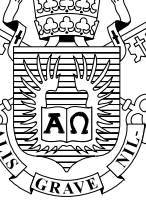

Henrique Bastos Graciosa

\section{A Transformação das Redes Ópticas no Acesso e no Anel Backbone Metropolitano: Alternativas Tecnológicas, Econômicas e Novos Serviços}

Dissertação de Mestrado apresentada como requisito parcial para obtenção do grau de Mestre pelo Programa de PósGraduação em Engenharia Elétrica do Departamento de Engenharia Elétrica do Departamento de Engenharia Elétrica do Centro Técnico Científico da PUC-Rio. Aprovada pela Comissão Examinadora abaixo assinada.

Prof. Marbey Manhães Mosso

Orientador

Centro de Estudos em Telecomunicalões - PUC-Rio

Profa. Claudia Barucke Marcondes Paes Leme

Marcondes Barucke Comunicação Pedagógica

Profa. Maria Cristina Ribeiro Carv alho

Centro de Estudos em Telecomunicações - PUC-Rio

Prof. Gláucio Lima Siqueira

Centro de Estudos em Telecomunicações - PUC-Rio

Prof. Rodolfo Araujo de Azevedo Lima

Instituto de Pesquisas da Marinha

Prof. José Eugenio Leal Coordenador Setorial do Centro

Técnico Científico - PUC-Rio

Rio de Janeiro, 24 de janeiro de 2012 
Todos os direitos reservados. É proibida a reprodução total ou parcial do trabalho sem autorização da universidade, do autor e do orientador.

\section{Henrique Bastos Graciosa}

Graduado em Engenharia Elétrica com ênfase em telecomunicações pela Universidade Estadual de Campinas (Unicamp) em 2001. Foi selecionado pela CAPES em 1999 para cursar parte de sua graduação no Institut National des Sciences Appliquées (INSA) de Lyon na França no ano 2000. Desde 2002 é colaborador da empresa Padtec S.A, empresa brasileira líder no desenvolvimento e fornecimento de equipamentos e soluções para o transporte óptico. Tendo atuado nas áreas de desenvolvimento de produtos opto-eletrônicos para telecomunicações e projetos de redes ópticas, atualmente é responsável pela área de vendas técnicas no Rio de Janeiro.

Ficha Catalográfica

Graciosa, Henrique Bastos

A transformação das redes ópticas no acesso e no anel backbone metropolitano: alternativas tecnológicas, econômicas e novos serviços / Henrique Bastos Graciosa ; orientador: Marbey Manhães Mosso. - 2012.

160 f. ; $30 \mathrm{~cm}$

Dissertação (mestrado) - Pontifícia Universidade Católica do Rio de Janeiro, Departamento de Engenharia Elétrica, 2012.

Inclui bibliografia

1. Engenharia elétrica - Teses. 2. ETHERNET. 3. Redes de acesso. 4. GPON. 5. XG-PON. 6. WDM-PON. 7. CAPEX. 8. OPEX. 9. Valor adicional. 10. ODN. 11. Redes metropolitanas. 12. DWDM. 13. ROADM. 14. OTN/G709. 15. TCO. I. Mosso, Marbey Manhães. II. Pontifícia Universidade Católica do Rio de Janeiro. Departamento de Engenharia Elétrica. III. Título. 


\section{Agradecimentos}

À Pontifícia Universidade Católica do Rio de Janeiro, por todo o suporte dispensado durante o curso e elaboração da dissertação.

Ao meu orientador Prof. Marbey Manhães Mosso, pela orientação, paciência, dedicação, estímulo e compreensão, sem os quais teria sido penosa a conciliação entre trabalho e estudo, sobretudo nas fases finais da elaboração da presente dissertação.

Aos professores com os quais tive aulas e aos colegas do CETUC/PUC.

Aos meus pais, Helio e Sandra, pela educação, valores e amor que deram as bases para eu atingir mais essa etapa de minha vida.

Aos meus irmãos, Guilherme e Marcelo, sempre presentes nos momentos importantes de minha vida.

Aos meus padrinhos Sergio e Gilda e avó Nely, pelo entendimento de meus momentos de reclusão e ausência nos encontros familiares na fase final do estudo.

A minha noiva Marcia Helena, cujo apoio, incentivo, serenidade e amor incondicionais me deram a tranquilidade de poder finalizar a presente dissertação.

Aos meus líderes na Padtec, Jorge Salomão e Argemiro, pela compreensão nos momentos de ausência e incentivo à conclusão do mestrado. 


\section{Resumo}

Graciosa, Henrique Bastos; Mosso, Marbey Manhães (Orientador). A Transformação das Redes Ópticas no Acesso e no Anel Backbone Metropolitano: Alternativas Tecnológicas, Econômicas e Novos Serviços. Rio de Janeiro, 2012. 160p. Dissertação de Mestrado - Departamento de Engenharia Elétrica, Pontifícia Universidade Católica do Rio de Janeiro.

A presente dissertação tem como objetivo avaliar as mudanças que estão ocorrendo nas arquiteturas das redes ópticas dos operadores de telecomunicações para adequarem-se aos novos serviços oferecidos aos usuários. Esses serviços demandam cada vez mais flexibilidade, confiabilidade e capacidade de todos os níveis das redes ópticas, desde o acesso até os backbones metropolitanos e de longa distância, passando pelas redes de agregação. No acesso, a principal transformação é a extensão da fibra óptica para pontos cada vez mais próximos do usuário, utilizando-se de tecnologia PON (Passive Optical Network), de maneira a oferecer maior banda permitindo a diversificação de serviços e aplicações, como vídeo de alta definição com interatividade. No backbone metropolitano também são verificadas importantes mudanças, implicando a necessidade de capacidades de transporte cada vez maiores (múltiplos lambdas de 40 Gbps ou 100 Gbps) com uso de DWDM (Dense Wavelength Division Multiplexing) e OTN (Optical Transport Network), com roteamento de lambda no nível óptico por meio do uso de ROADM (Reconfigurable Add-Drop Multiplexer) e switching eletrônico também suportado pela OTN. Essa rede de elevada capacidade conecta-se diretamente às redes de acesso ou redes coletoras ou de agregação, aptas a transportar de forma eficiente tráfego baseado em pacotes. Nesse contexto, substituem-se as soluções tradicionais baseadas em TDM (Time Division Multiplexing) por soluções mais adaptadas ao transporte de pacotes e que garantam qualidade de serviço. Essa mesma rede de alta capacidade metropolitana também deve fazer interface com as redes de transmissão regionais e interurbanas de longa distância. $\mathrm{O}$ trabalho dará especial atenção às topologias de rede de acesso e backbone metropolitano descritas acima, comparando arquiteturas e investimentos associados.

\section{Palavras-chave}

ETHERNET, Redes de Acesso, GPON, XG-PON, WDM-PON, CAPEX, OPEX, Valor Adicional, ODN, Redes Metropolitanas, DWDM, ROADM, OTN/G709,TCO. 


\section{Abstract}

Graciosa, Henrique Bastos; Mosso, Marbey Manhães (Advisor). Optical Network Transformation on Access and Metropolitan Backbone Ring: Technological and Economical Alternatives and New Services. Rio de Janeiro, 2012. 160p. MSc. Dissertation - Departamento de Engenharia Elétrica, Pontifícia Universidade Católica do Rio de Janeiro.

This dissertation aims to evaluate the changes that are occurring in the telecom operators' optical network infrastructures to suit to the new services offered to their customers. These services demand increasingly flexibility, reliability and capacity from all optical network layers, from access to long-haul and metropolitan backbone, including aggregation layer. On access, the main transformation is the extension of the optical fiber to points closer to the customers using PON (Passive Optical Networks) technologies in order to offer higher bandwidths. With this strategy it is possible to enable applications and services diversification, like interactivity high definition video. It is also verified important changes on the metropolitan backbone. It is observed the need of improving the transport capacity (multiple 40 Gbps or 100 Gbps wavelengths) using DWDM (Dense Wavelength Division Multiplexing) and OTN (Optical Transport Network), with wavelength routing using ROADM (Reconfigurable Add-Drop Multiplexer) and electronic switching. This high-capacity network is connected directly to the access or aggregation networks, which are able to efficiently carry packet-based traffic. In this context, traditional solutions based on TDM (Time Division Multiplexing) are replaced by solutions best adapted for packet transport with reliable quality of service. This same high-capacity metropolitan network should also be connected with the regional and longhaul networks. This work will give special attention to the access network topologies and metro backbone described above, comparing architectures and related investments.

\section{Keywords}

ETHERNET, Access Networks, GPON, XG-PON, WDM-PON, CAPEX, OPEX, Value Added, ODN, Metropolitan Networks, DWDM, ROADM, OTN/G709,TCO. 


\section{Sumário}

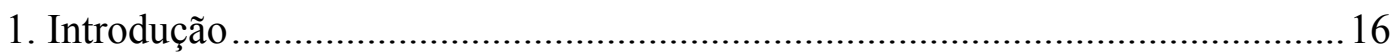

2. Perspectivas de Consumo de Banda no Acesso..................................................20

2.1. Novos Serviços e Tecnologias de Acesso Banda Larga ................................. 20

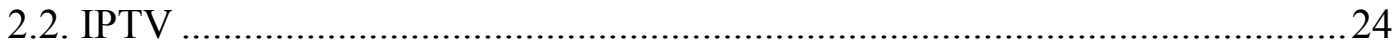

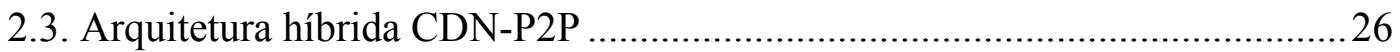

2.4. Comentários e Conclusões do Capítulo .............................................................2 28

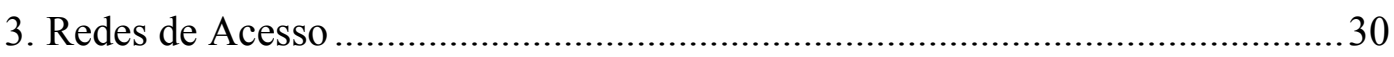

3.1. Tecnologias de Acesso Banda Larga para Suporte aos Novos Serviços..........30

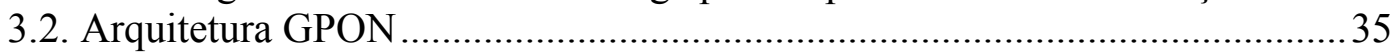

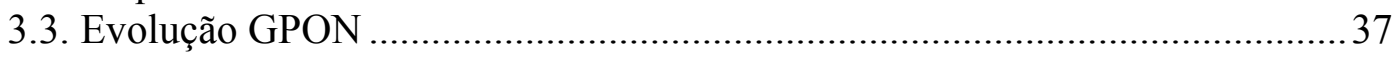

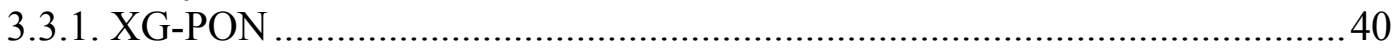

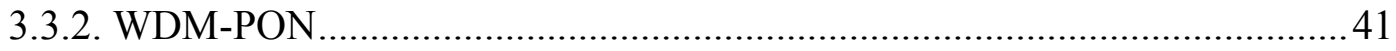

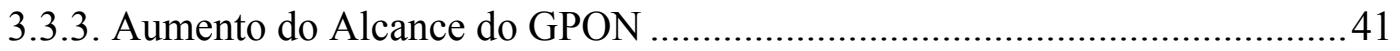

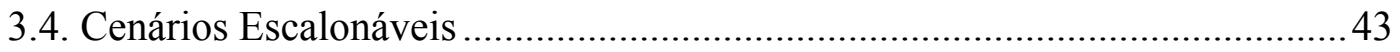

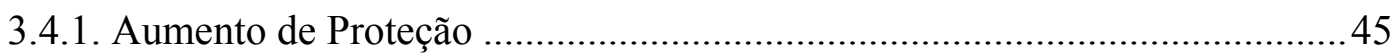

3.4.2. Aumento de Banda - Cenário 1: XG-PON Assimétrico ou Simétrico.......... 47

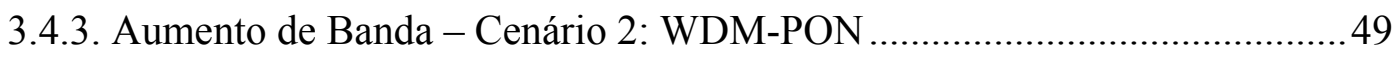

3.4.4. Evolução GPON com Coexistência - Cenário 1: Overlaid-PON ..................51

3.4.5. Evolução GPON com Coexistência - Cenário 2: Overlaid-PON com

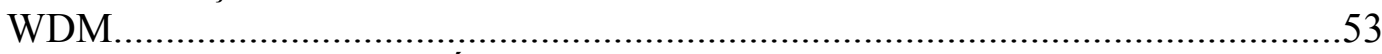

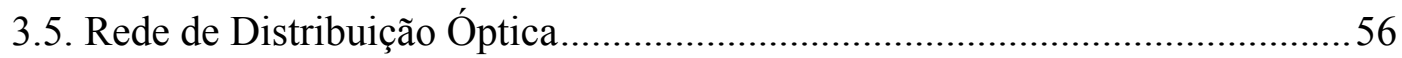

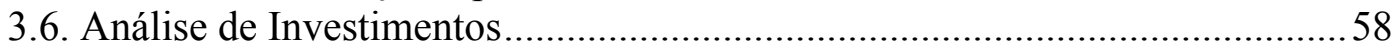

3.7. Comentários e Conclusões do Capítulo .......................................................... 70

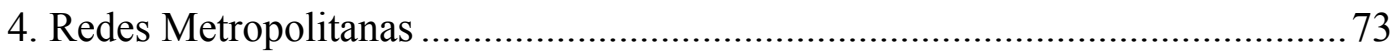

4.1. Levantamento de Capacidade Requerida pela Rede Metropolitana................ 75

4.1.1. Banda larga FTTX utilizando rede GPON ............................................... 75

4.1.2. Conexão para grandes clientes corporativos, SAN (Storage Area

Network), conexão com outras operadoras e carriers e backhauling celular.........80

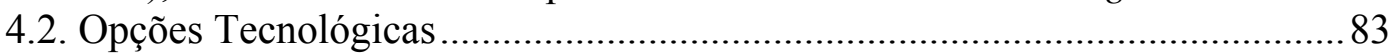

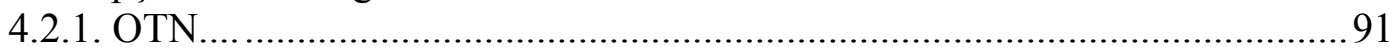

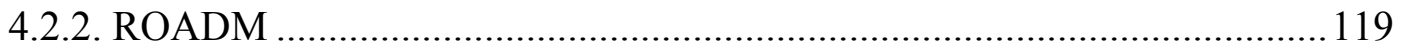

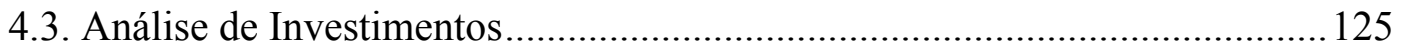

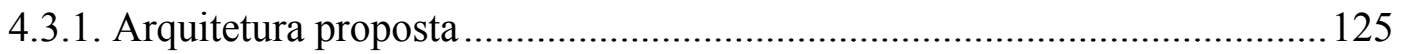

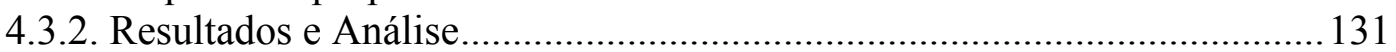

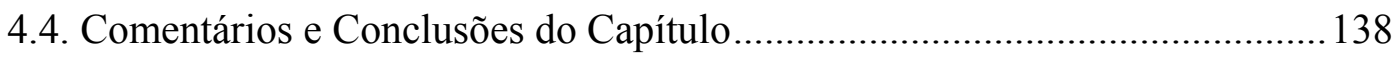

5. Conclusões e Comentários finais................................................................... 141

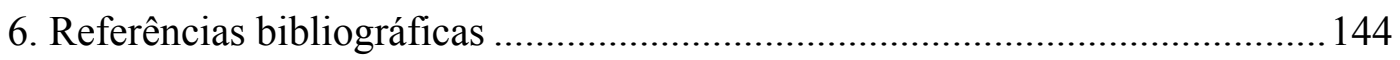




\section{Lista de tabelas}

Tabela 1 - Tráfego de downstream e upstream para distintas aplicações. .............2 21

Tabela 2 - Fases de evolução do consumo de banda com vídeo. ........................... 22

Tabela 3 - Quantidade de usuários suportados por cada classe de ODN para

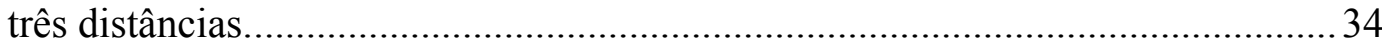

Tabela 4 - Comparação entre tecnologias de amplificação para extensão de

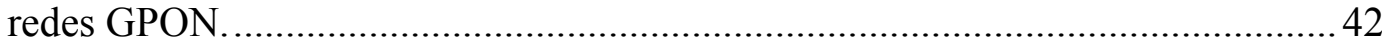

Tabela 5 - Comparação entre banda oferecida pelo GPON e pelo XG-GPON..... 49

Tabela 6 - Premissa para prospecção de banda requerida pela rede metropolitana para atendimento a serviços banda larga com FTTH..................... 75

Tabela 7 - Cenários considerados na análise...................................................... 76

Tabela 8 - Banda total requerida na rede metropolitana para cada cenário........... 76

Tabela 9 - Estrutura OTN para transporte de distintos tipos de sinais clientes de acordo a versão da recomendação G.709 de 2001............................................ 116

Tabela 10 - Estrutura OTN para transporte de distintos tipos de sinais clientes de acordo a recomendação G.709 de 2009....................................................... 117

Tabela 11 - Composição da infraestrutura do nó com e sem ROADM............... 130

Tabela 12 - Cenários considerados nas análises de investimentos......................131

Tabela 13 - Configuração de equipamentos OLT e ONT utilizada na seção 3.6.

Tabela 14 - Configuração de equipamentos correspondentes aos cenários

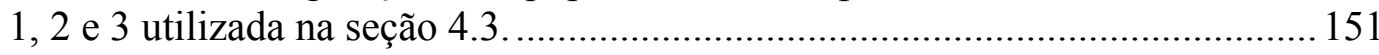

Tabela 15 - Configuração de equipamentos correspondentes aos cenários 4,5 e 6 utilizada na seção 4.3 .

Tabela 16 - Configuração de equipamentos correspondentes aos cenários 7 e 8 utilizada na seção 4.3 


\section{Lista de figuras}

Figura 1 - Visão geral dos tipos de redes e aplicações com destaque ao backbone metropolitano e ao acesso FTTH.

Figura 2 - Comparação entre a banda consumida para distintos serviços e a banda oferecida por distintas tecnologias de acesso banda larga........................ 23

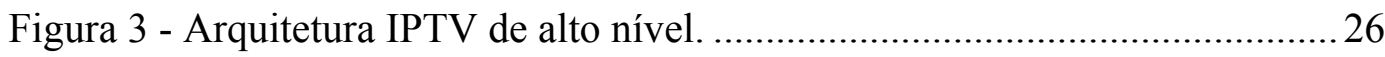

Figura 4 - Etapas potenciais de evolução dos serviços IPTV................................ 26

Figura 5 - Arquitetura para VoD proposta em "A Hybrid CDN-P2P system for

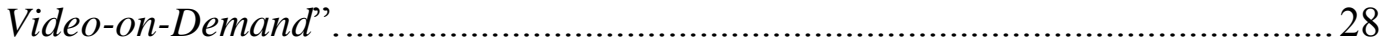

Figura 6 - Arquitetura de uma rede PON genérica.............................................. 32

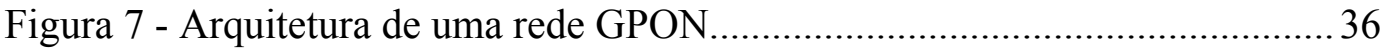

Figura 8 - Arquitetura de uma rede GPON com suporte a vídeo. ......................... 37

Figura 9 - Evolução da rede GPON em função do tempo...................................... 39

Figura 10 - Plano de comprimento de onda de acordo com as recomendações

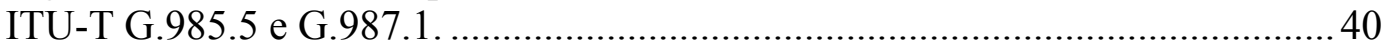

Figura 11 - Topologia exemplificando arquitetura para aumento do alcance da

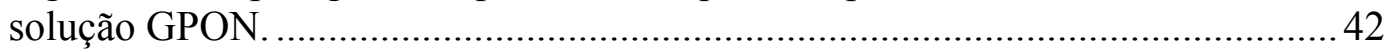

Figura 12 - Exemplo de arquiteturas FTTX. ....................................................... 44

Figura 13 - Topologia do cenário básico GPON ................................................ 45

Figura 14 - Proteção do cabo localizado entre a OLT e o primeiro divisor óptico, utilizando-se um comutador óptico.

Figura 15 - Proteção da interface da OLT e do cabo localizado entre a OLT e o primeiro divisor óptico.

Figura 16 - Proteção da interface da OLT, do cabo localizado entre a OLT e o primeiro divisor óptico e dos cabos de acesso a ONT......................................... 47

Figura 17 - Topologia XG-PON substituindo topologia básica GPON. ............... 48



Figura 19 - Topologia XG-PON coexistindo com topologia básica GPON.......... 52

Figura 20 - Topologia WDM-PON coexistindo com topologia XG-PON e

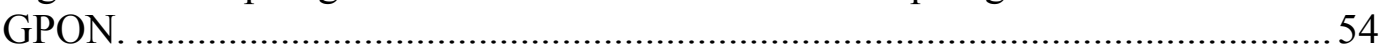

Figura 21 - Detalhamento do Filtro e Multiplexador/Demultiplexador

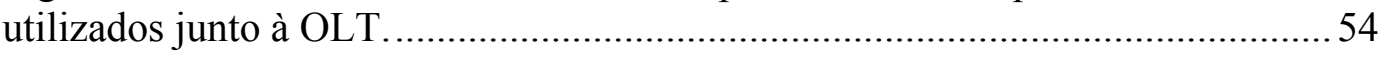

Figura 22 - Detalhamento do Filtro, divisor óptico e Multiplexador/

Demultiplexador utilizados ao longo da rede de distribuição óptica.....................55

Figura 23 - Arquitetura ODN com dois níveis de distribuição. ............................ 56

Figura 24 - Arquitetura ODN com um nível de distribuição.................................56 
Figura 25 - Configuração OLT mostrando conexões com rede de acesso e

Figura 26 - Valor adicional de elementos passivos para preparação da rede para suporte à ampliação em relação ao valor total de Capex de equipamentos considerando uma única rede com 32 usuários.

Figura 27 - Valor adicional de elementos passivos para preparação da rede para suporte à ampliação em relação ao valor total de Capex de equipamentos considerando 64 redes com 32 usuários cada.

Figura 28 - Valor adicional de elementos passivos para preparação da rede para suporte à ampliação em relação ao valor total de Capex de equipamentos e ODN considerando uma única rede com 32 usuários.

Figura 29 - Valor adicional de elementos passivos para preparação da rede para suporte à ampliação em relação ao valor total do Capex de equipamentos e ODN considerando 64 redes com 32 usuários cada.

Figura 30 - Capex adicional em relação ao Capex total para preparação da rede para suporte aos cenários 1 e 2 em função da variação do comprimento médio de cada rede GPON para distintos 3 valores de referência do $\mathrm{km}$ de ODN instalada para 1 rede com 32 usuários.

Figura 31 - Capex adicional em relação ao Capex total para preparação da rede para suporte aos cenários 1 e 2 em função da variação do comprimento médio de cada rede GPON para distintos 3 valores de referência do $\mathrm{km}$ de ODN instalada para 64 redes com 32 usuários cada.

Figura 32 - Estrutura posicionada junto a OLT pronta para receber o cenário 2. . 69

Figura 33 - Estrutura posicionada na ODN pronta para receber o cenário 2

Figura 34 - Visão geral das redes metropolitanas ilustrando suas conexões e diversidades de atendimentos.

Figura 35 - Diversidade de protocolos e velocidades na agregação e capacidades de transporte exigidas das redes metropolitanas. 82

Figura 36 - Sistema de transmissão WDM genérico............................................. 86

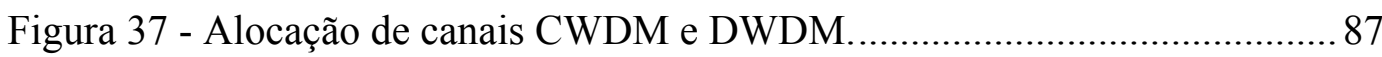

Figura 38 - Diagrama de um sistema de transmissão DWDM genérico mostrando seus elementos principais e a conexão entre eles.

Figura 39 - Evolução nos padrões e tecnologias para as redes de transporte........ 92

Figura 40 - Representação da estrutura de camadas............................................9 94

Figura 41 - Representação da estrutura de camadas simplificada........................ 95

Figura 42 - Estrutura do quadro do canal óptico (OCh) ...................................... 95

Figura 43 - Estrutura do quadro OTN detalhada..................................................96

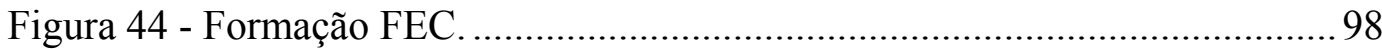

Figura 45 - Algumas opções de mapeamento e multiplexação...........................100

Figura 46 - BER corrigida em relação à potência de recepção para situação com e sem FEC. 
Figura 47 - Relação teórica entre BER de entrada e BER de saída com FEC utilizando RS $(255,239)$ de acordo com a ITU-T G.975 ................................. 102

Figura 48 - Exemplo de solução com e sem uso de multiplexação OTN............ 104

Figura 49 - Estrutura de mapeamento com uso de SDH. ................................... 106

Figura 50 - Estrutura de mapeamento sem uso de SDH..................................... 107

Figura 51 - Comparação de facilidades de gerenciamento em sistema com e sem gerenciamento OTN.

Figura 52 - Comparação entre etapas para identificação de falha ou degradação com e sem gerência OAM OTN.

Figura 53 - Conexão Gigabit Ethernet ou 10 Gigabit Ethernet entre nós C e E com transporte feito diretamente sobre OTN.

Figura 54 - Sistema WDM com conexão (lambda) dedicada para cada atendimento.

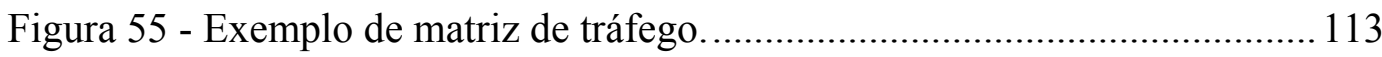

Figura 56 - Sistema WDM OTN para transporte de sub-lambda....................... 113

Figura 57 - Gerenciamento de sinal cliente em ambiente multi-domínio

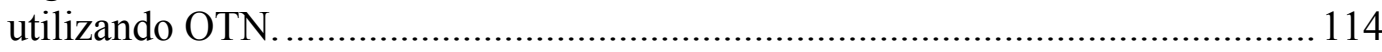

Figura 58 - Redes ópticas com ROADMs de distintos graus ............................. 120

Figura 59 - Detalhamento de nó com ROADM grau 4..................................... 120

Figura 60 - Detalhamento de nó ROADM grau 3 directionless.......................... 123

Figura 61 - Conceito da arquitetura de rede óptica metropolitana DWDM

OTN utilizada na análise de investimentos. ................................................... 126

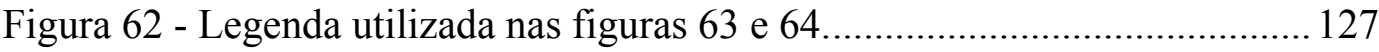

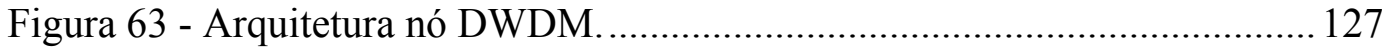

Figura 64 - Arquitetura nó DWDM com ROADM........................................ 128

Figura 65 - Capex para ativação da infraestrutura de cada cenário em relação

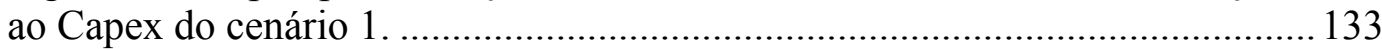

Figura 66 - TCO em 5 anos para ativação completa de cada cenário em relação ao TCO do cenário 1 .

Figura 67 - Capacidade, TCO em 5 anos e custo do Mbps de cada cenário em relação aos mesmos parâmetros do cenário 1......................................................... 136

Figura 68 - TCO em 5 anos e custo do Mbps dos cenários 5, 6, 7 e 8 em relação aos mesmos parâmetros do cenário 1 com análise de sensibilidade. 


\section{Lista de Acrônimos}

ADM - Add and Drop Multiplexer

ADM - Add and Drop Multiplexer

ADSL - Asymmetric Digital Subscriber Line

ADSL2 - Asymmetric Digital Subscriber Line 2

ADSL2+- Asymmetric Digital Subscriber Line 2 Plus

AMP - Asynchronous Mapping Procedure

ANSI - American National Standards Institute

ATM - Asynchronous Transfer Mode

AWG - Arrayed Waveguide Grating

BER - Bit Error Rate

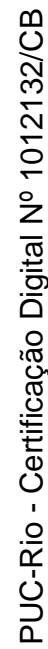

BMP - Bit-Synchronous Mapping Procedure

B-RAS - Broadband Remote Access Servers

BSS - Business Support Systems

Capex - Capital Expediture

CATV - Cable TV

CDC - Colorless, Directionless, Contentioless

CDN - Content Distribution/Delivery Network

CDN-P2P - Content Distribution Network Peer-to-Peer

CWDM - Coarse Wavelength Division Multiplexing

DBA - Dynamic Bandwidth Assignment

DCF - Dispersion Compensating Fiber

DFB - Distributed Feedback

DGO - Distribuidor Geral Óptico

DMB - Digital Multimedia Broadcast

DOCSIS - Data Over Cable Service Interface Specification

DSL - Digital Subscriber Line

DSLAMs - Digital Subscriber Line Access Multiplexers

DVB-H - Direct Video Broadcast - Handset

DWDM - Dense Wavelength Division Multiplexing

EDFA - Erbium-Doped Fiber Amplifier 
EOAM - Ethernet Operation, Administration and Maintenance

EPON - Ethernet Passive Optical Network

ESCON - Enterprise Systems Connection

FAT - Fiber Access Terminal

FC - Fiber Channel

FDM - Frequency Division Multiplexing

FDT - Fiber Distribution Terminal

FEC - Forward Error Correction

FICON - Fiber Connection

FSAN - Full Service Access Network

FTTH - Fiber-to-the-Home

GbE - Gigabit Ethernet

Gbps - Gigabits per second

GFC - Gigabit Fiber Channel

GFP-F - Frame-Mapped Generic Framing Procedure

GPON - Gigabit Capable Passive Optical Network

HD - High-Definition

HD-SDI - High-Definition Serial Digital Interface

HDTV - High-Definition Television

HFC - Hybrid Fiber-Coaxial

IEEE - Institute of Electrical and Electronics Engineers

IP - Internet Protocol

IPTV - Internet Protocol Television

ITU-T - International Telecommunication Union - Telecommunication

Standardization Sector

$\mathrm{km}$ - quilômetro

LAN - Local Area Network

MAN - Metropolitan Area Network

Mbps - Megabits per seconds

MPLS - Multiprotocol Label Switching

MPLS-TP - Multiprotocol Label Switching - Transport Profile

NG-PON - Next Generation - Passive Optical Network

$\mathrm{nm}$ - nanômetro

OADM - Optical Add-Drop Multiplexer 
OAM - Operation, Administration and Maintenance

OCh/OChr - Optical Channel

ODN - Optical Distribution Network

ODU - Optical Data Unit

OLT - Optical Line Terminator

OMS - Optical Multiplex Section

ONT - Optical Network Terminal

ONU - Optical Network Unit

Opex - Operational Expenditure

OPS - Optical Physical Section

OPU - Optical Payload Unit

OSI - Open Systems Interconnection

OSS - Operational Support Systems

OTN - Optical Transport Network

OTS - Optical Transmission Section

OTU - Optical Channel Transport Unit

P2P - Peer-to-Peer system

PLC - Planar Lightwave Circuit

PMD - Polarization Mode Dispersion

PON - Passive Optical Network

P-OTS - Packet-Optical Transport System

QoE - Quality of Experience

QoS - Quality of Service

RF - Radio Frequency

ROADM - Reconfigurable Add-Drop Multiplexer

RPT - Remote Protocol Termination

SAN - Storage Area Network

SD - Standard-Definition

SDH - Synchronous Digital Hierarchy

SDI - Serial Digital Interface

SD-SDI - Standard-Definition Serial Digital Interface

SDTV - Standard-Definition Television

SMF - Single-Mode Optical Fiber

SMPTE - Society of Motion Picture and Television Engineers 
SNIA - Storage Network Industry Association

STM - Synchronous Transport Module

Tbps - Terabits per second

TCM - Tandem Connection Monitoring

TCO - Total Cost of Ownership

TDM - Time Division Multiplexing

TS - Timeslot

UHDTV - Ultra High-Definition Television

VDSL - Very High Speed Digital Subscriber Line

VDSL2 - Very High Speed Digital Subscriber Line 2

VoD - Video on Demand

VoIP - Voice over Internet Protocol

WB - Wavelength Blocking

WDM - Wavelength Division Multiplexing

WDM-PON - Wavelength Division Multiplexer - Passive Optical Network

WFB - Wavelength Blocking Filter

WiMAX - Worldwide Interoperability for Microwave Access

WSS - Wavelength Selective Switch

XG-PON - Ten Gigabit - Passive Optical Network 